\title{
Cytologic analysis of the mammary papillar discharge in a canine micropapillary carcinoma
}

\author{
Geovanni Dantas Cassali ${ }^{1}$, Lidianne Narducci Monteiro ${ }^{1}$, Conrado de Oliveira Gamba ${ }^{1}$, \\ Karine Araújo Damasceno ${ }^{1}$, Cecília Bonolo de Campos $^{1,2}$, Breno Souza Salgado ${ }^{3}$ \\ ${ }^{1}$ Laboratory of Comparative Pathology, Department of General Pathology, Biological Sciences Institute, Federal University of Minas Gerais (UFMG), \\ Belo Horizonte, Brazil; ${ }^{2}$ Department of Veterinary Clinic and Surgery, School of Agricultural and Veterinary Sciences, São Paulo State University \\ (FCAV/UNESP), Jaboticabal, Brazil; and ${ }^{3}$ University José do Rosário Vellano (UNIFENAS), Alfenas, Brazil
}

\section{Key Words}

Fine-needle aspiration, mammary neoplasms, mammary papilla, micropapillary neoplasms

\section{Correspondence}

G.D. Cassali, Laboratório de Patologia Comparada, Departamento de Patologia Geral, Instituto de Ciências Biológicas (ICB), Universidade Federal de Minas Gerais, Avenida Presidente Antônio Carlos, 6627, Belo Horizonte, Minas Gerais 31270-901, Brasil E-mail: cassalig@icb.ufmg.br

DOI:10.1111/vcp.12269

\begin{abstract}
This is a report on the cytologic analysis of the mammary papillar discharge in a 7-year-old female Doberman dog with an invasive micropapillary carcinoma. Cytologic evaluation of nipple discharge is a well-known method for the rapid diagnosis of breast cancer in women. However, there is no previous report regarding the use of this technique for assessing mammary tumors in dogs. The aim of this study was to describe the use of mammary papillar discharge cytology for diagnosing a micropapillary carcinoma in a dog. Cytologically, evaluation of the papillar discharge revealed cells arranged in clusters in a papillary pattern or in a morula-like arrangement, suggesting the diagnosis of a micropapillary carcinoma, which was subsequently confirmed by histopathology. Thus, mammary papillar discharge cytology should be considered as an ancillary method for evaluating mammary diseases in dogs.
\end{abstract}

\section{Case Presentation}

A 7-year-old female Doberman dog was admitted to the Veterinary School Teaching Hospital of the Federal University of Minas Gerais, Belo Horizonte, Brazil, with a mammary tumor characterized by a nonulcerated, nonalopecic, plaque-like, firm subcutaneous mass extending from the right caudal abdominal to the inguinal mammary glands with indistinct borders. The mass grew rapidly, was located in the dermis and subcutaneous tissue, and was adherent to the adjacent tissues. The presence of a serous papillar discharge was observed. The discharge and fine-needle aspirates (FNA) from the primary tumor were collected with a $25 \times 7 \mathrm{~mm}$ needle and smeared onto glass slides. Smears were air-dried, methanol-fixed, and stained using a Giemsa-type stain (Merck, Darmstadt, Germany).

Cytologic evaluation of the mammary papillar discharge revealed samples with moderate cellularity composed of epithelial cells arranged in clusters in a papillary (Figure 1A) or morule-like pattern (Figure 1B). Cells had a moderate amount of basophilic cytoplasm with distinct borders and round to oval nuclei with coarse chromatin and one or 2 prominent nucleoli. Moderate anisocytosis and anisokaryosis, high nuclear/cytoplasmic ratio, nuclear molding, binucleate cells and presence of foamy macrophages were also observed. The FNA sample was highly cellular, with numerous large epithelial cells arranged in a papillary fashion or in morule-like arrangements (Figure 2), and composed of similar neoplastic epithelial cells as described in the papillar discharge. In addition, isolated round to oval cells were detected. They had nuclei with irregularly distributed chromatin with one or 2 prominent nucleoli. The cytologic diagnosis was that of a malignant epithelial neoplasm. Given the morphologic features of the neoplastic cells described above and the anatomic location of the tumor, a presumptive diagnosis of a micropapillary mammary carcinoma was made.

The tumor was surgically excised in conjunction with a superficial inguinal lymph node. Grossly, the surgical specimen was characterized as a plaque-like, white, and somewhat lobulated mass weighing $75.70 \mathrm{~g}$ and measuring $14.7 \times 6.3 \times 2.0 \mathrm{~cm}$. Tissue samples were fixed in formalin and routinely processed. Subsequently, $4-\mu \mathrm{m}$ sections were cut and 


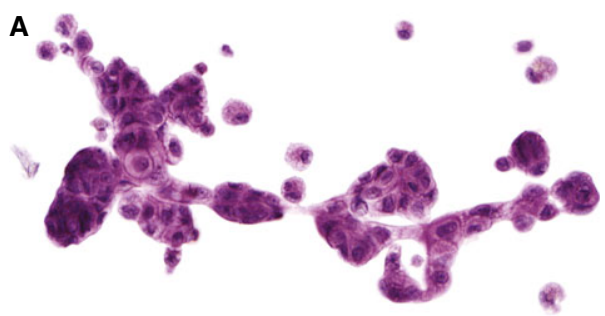

B
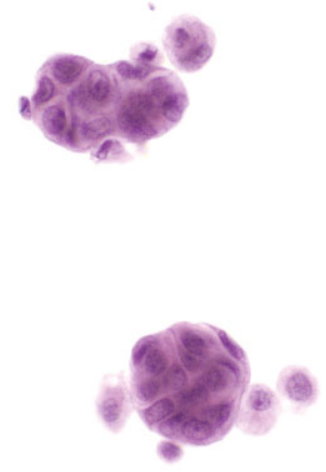

Figure 1. Smear of mammary papillar discharge of a dog with a mammary tumor. (A) Clusters of epithelial cells in a micropapillary pattern. Giemsa, $\times 40$ objective. (B) Morule- like clusters of neoplastic cells. Giemsa, $\times 40$ objective.

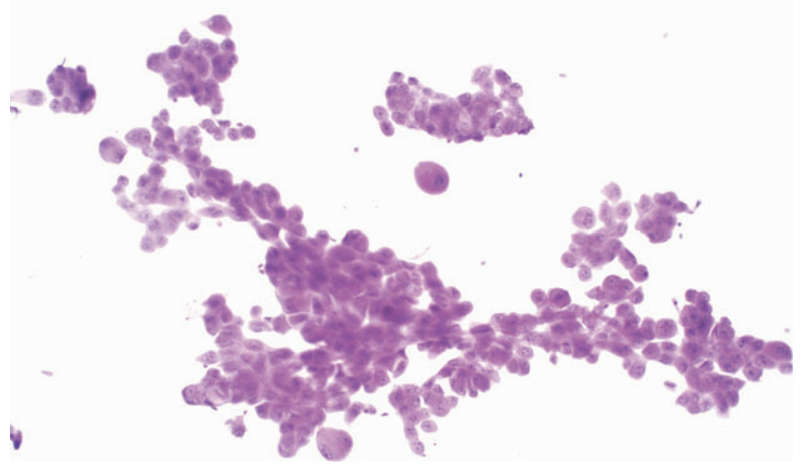

Figure 2. Smear of a fine-needle aspirate obtained from a mammary tumor in a dog. Clusters of epithelial cells in a micropapillary pattern similar to those seen in the papillar discharge cytology were observed. Giemsa, $\times 20$ objective.

stained with hematoxylin and eosin for microscopic evaluation.

Histologically, the neoplasm was characterized by clusters of epithelial cells without central fibrovascular cores. Such clusters were separated from the extracellular matrix by empty clear (cystic) spaces with the cellular apical surface polarized toward the outside. Some of these cystic spaces resembled lymphatic vessel-like channels and were filled with epithelial cells exhibiting a micropapillary pattern. Epithelial cells arranged in a similar histologic pattern were observed within mammary ducts (Figure 3A,B). In addition, morule-like clusters of epithelial cells were observed in the cortex and medulla of the inguinal lymph node. The presumptive diagnosis of micropapillary carcinoma made by cytology was confirmed by histopathology. Consequently, a final diagnosis of an invasive micropapillary carcinoma of the mammary gland with neoplastic cells within the mammary papilla discharge was made.

\section{Discussion}

Cytologic examination of the papillar discharge is an easy-to-perform, noninvasive, highly reliable diagnostic test for patients with breast lesions. ${ }^{1}$ How-
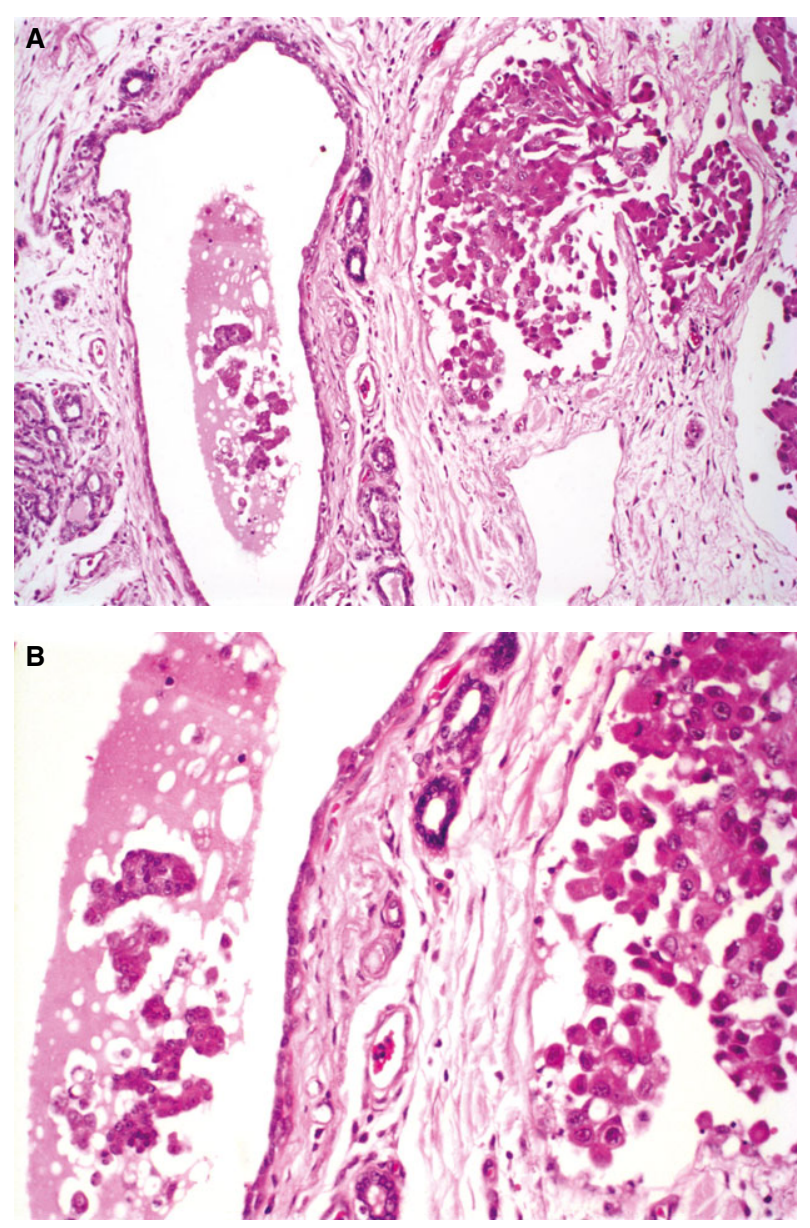

Figure 3. Histologic section of a mammary micropapillary carcinoma in a dog. (A) Irregular cystic formations with epithelial cells arranged in a micropapillary pattern. The same cells can be observed within the mammary duct. H\&E, $\times 20$ objective. (B) Higher magnification providing a detailed view of the characteristic features of micropapillary carcinoma in the cell clusters within the mammary duct. H\&E, $\times 40$ objective. 
ever, despite the high frequency of mammary lesions in dogs, there is no report describing the use of this method for assessing mammary lesions. There is only one report describing the use of this technique in a case of dirofilariasis diagnosed by papillar discharge cytology in a bitch. ${ }^{2}$

In women, nipple discharge cytology (the name used for papillar discharge cytology in women) is classified as physiologic or pathologic. Physiologic discharge is usually bilateral and originating from several ducts. The most common etiologies include pregnancy, lactation, hypothyroidism, pituitary adenoma, oral contraceptives, antihypertensives, and tranquilizers. Pathologic nipple discharge is often not related to lactation, it is usually persistent and typically unilateral, involving just a single duct. ${ }^{3}$ Nipple discharge can have variable characteristics, including a milky or multicolored appearance, and sticky, purulent, clear, and watery, yellow or serous, pink or serosanguineous, and sanguineous consistency. These characteristics may also assist in the early diagnosis and management of mammary gland disease. ${ }^{4}$

In this study, the cytologic characteristics observed in smears of mammary papillar discharge were similar to those reported for canine micropapillary carcinomas obtained by fine-needle aspiration in dogs. ${ }^{5,6}$ Canine mammary invasive micropapillary carcinoma is a morphologically distinctive type of invasive carcinoma of the mammary gland of dogs that has recently been described. ${ }^{7,8}$ The tumor is characterized by the presence of tumor cells in morula-like clusters inside cystic spaces. This histologic type has been associated with lymph node metastases and a poor prognosis in both women ${ }^{9}$ and dogs. ${ }^{8,10}$ In the present case, the characteristic morphologic features allowed the diagnosis of the neoplasm by evaluating the mammary papillar discharge.

Criteria for nipple discharge cytology have been well defined, but there is a certain morphologic overlap of features between benign and malignant entities. ${ }^{11}$ In this case, the cytologic diagnosis of malignancy was possible and later confirmed by histopathology, indicating that mammary papillar discharge cytology can provide a reliable diagnosis. However, as this is the first report on one case, it is not possible to make further assumptions regarding the differentiation of benign and malignant lesions in dogs using just cytology.

According to some authors ${ }^{12}$, the observation of epithelial cell groups in nipple discharge cytology is strongly suggestive of neoplastic disease. Pathologic nipple discharge is most commonly caused by benign conditions like intraductal papillomas, duct ectasia, papillomatosis, mastitis, and fibrocystic changes. ${ }^{3,4}$ The evaluation and diagnosis of the discharge is important for the early detection of carcinoma, when present; and in the case of benign disease, it is necessary to stop the incommodious discharge. ${ }^{4}$ The majority of women with breast cancer who manifest with isolated nipple discharge have an early stage disease associated with in situ ductal carcinoma. ${ }^{13}$

Previous studies have demonstrated important disagreement regarding the sensitivity, specificity, and accuracy of nipple discharge cytology in women. ${ }^{1,14}$ The most common factor that led to false-negative results in cytology was the insufficient cellularity of specimens. ${ }^{14}$ Nipple discharge cytology alone was highly reliable in identifying $92.3 \%$ of all atypical and/ or malignant lesions and $69.6 \%$ of benign intraductal papillomas, so the vast majority of worrisome lesions were identified by cytology and these findings directed surgeons to further investigation, indicating the diagnostic value of this method. ${ }^{1}$ A lower sensitivity has been attributed to nipple discharge when compared to FNA analysis in women, particularly because neoplastic cells tend to decrease or disappear in the nipple discharge. This lack of cellularity can make a reliable diagnosis impossible. ${ }^{15}$ However, practically no significant difference in specificity has been demonstrated ${ }^{16}$, while the percentage of false-negative results was higher in comparison to false-positive results. ${ }^{17}$

The College of American Pathologists Interlaboratory Comparison Program in Nongynecologic Cytopathology is skeptical concerning the usefulness of spontaneous nipple discharge cytology. ${ }^{11}$ As in many other types of cytologic assessments, a cytologic diagnosis of benign nipple discharge cytology does not exclude malignancy, and any suspicion for a malignant cell population requires confirmation of the malignancy of a process in the mammary gland prior to definitive patient management. ${ }^{11}$ Nevertheless, despite the possibility of not confirming malignancy, nipple discharge can help in the differentiation of neoplastic disease from other conditions, such as infections. In dogs, considering the high frequency both of mammary tumors and mammary inflammation due to infection, cytologic evaluation of mammary papillar discharge may be a new method complementing the routinely used FNA and histopathologic examination of the mammary gland.

\section{Conclusions}

Cytologic analysis of mammary papillar discharge represents an uncomplicated diagnostic method that 
may be implemented as an ancillary technique for diagnosing mammary lesions, including mammary carcinoma. This technique is largely used in women with breast lesions. However, there are no studies regarding the use of mammary papillar discharge for diagnostic purpose in canine mammary gland tumors. Therefore, further studies are encouraged to assess the usefulness of this technique in dogs.

\section{Acknowledgments}

This work was financially supported by Fundação de Amparo à Pesquisa de Minas Gerais (FAPEMIG), Conselho Nacional de Desenvolvimento Científico e Tecnológico (CNPq), and Coordenação de Aperfeiçoamento de Pessoal de Nível Superior (CAPES), Brazil.

\section{References}

1. Kalu ON, Chow C, Wheeler A, Kong C, Wapnir I. The diagnostic value of nipple discharge cytology: breast imaging complements predictive value of nipple discharge cytology. J Surg Oncol. 2012;106:381-385.

2. Manuali E, Eleni C, Giovannini P, Costarelli S, Ciorba A. Unusual finding in a nipple discharge of a female dog: dirofilariasis of the breast. Diagn Cytopathol. 2005;32:108-109.

3. Zervoudis S, Iatrakis G, Economides P, Polyzos D, Navrozoglou I. Nipple discharge screening. Womens Health. 2010;6:135-151.

4. Hussain AN, Policarpio C, Vincent MT. Evaluating nipple discharge. Obstet Gynecol Surv. 2006;61:278-283.

5. Cassali GD, Gärtner F, Vieira da Silva MJ, Schmitt FC. Cytological diagnosis of a metastatic canine mammary tumor in pleural effusion. Arq Bras Med Vet Zootec. 1999;51:307-310.

6. Salgado BS, Monteiro LN, Colodel MM, et al. Clinical, cytologic, and histologic features of a mammary micro- papillary carcinoma in a dog. Vet Clin Pathol. 2013;42:382-385.

7. Cassali GD, Serakides R, Gärtner F, Schmitt FC. Invasive micropapillary carcinoma of the dog mammary gland. A case report. Arq Bras Med Vet Zootec. 2002;54:366-369.

8. Gamba CO, Dias EJ, Ribeiro LG, et al. Histopathological and immunohistochemical assessment of invasive micropapillary mammary carcinoma in dogs: a retrospective study. Vet J. 2013;196:241-246.

9. Paterakos M, Watokin WG, Edgerton SM, Moore DH, Thor AD. Invasive micropapillary carcinoma of the breast: a prognostic study. Hum Pathol. 1999;30:1459-1463.

10. Cassali GD, Lavalle GE, de Nardi AB, et al. Consensus for the diagnosis, prognosis, and treatment of canine mammary tumors. Braz J Vet Pathol. 201 1;4:153-180.

11. Moriarty AT, Schwartz MR, Laucirica R, et al. Cytology of Spontaneous Nipple Discharge - Is ItWorth It? Performance of Nipple Discharge Preparations in the College of American Pathologists Interlaboratory Comparison Program in Nongynecologic Cytopathology. Arch Pathol Lab Med. 2013;137:1039-1042.

12. Saphir O. Cytologic examination of breast secretion. Am J Clin Pathol. 1950;20:1001-1012.

13. Cabioglu N, Krishnamurthy S, Kuerer HM, et al. Feasibility of breast-conserving surgery for patients with breast carcinoma associated with nipple discharge. Cancer. 2004;101:508-517.

14. Kaplan R, Hoda SA, Hoda RS. Cytological evaluation of bloody nipple discharge fluid. Diagn Cytopathol. 2013;41:1833-185.

15. Halbe HW. Tratado de Ginecologia [Treaty Gynecology]. São Paulo, SP: Roca; 1993:773.

16. Lee WY. Cytology of abnormal nipple discharge: a cytohistological correlation. Cytopathol. 2003;14:19-26.

17. Takeda T, Matsui A, Sato Y, et al. Nipple discharge cytology in mass screening for breast cancer. Acta Cytol. 1990;34:161-164. 